\title{
Enhanced Corticospinal Excitability in the Tibialis Anterior During Static Stretching of the Soleus in Young Healthy Individuals
}

Francesco Budini ( $\square$ francesco.budini@gmail.com )

University of Graz

Christova Monica

Universities of Applied Sciences Joanneum

\section{Research Article}

Keywords: Static Stretching, Cortico spinal excitability, Motor Evoked Potential

Posted Date: December 6th, 2021

DOI: https://doi.org/10.21203/rs.3.rs-1110694/v1

License: (c) (i) This work is licensed under a Creative Commons Attribution 4.0 International License.

Read Full License 


\section{Abstract}

Corticospinal excitability is known to be affected by afferent inflow arising from the proprioceptors during active or passive muscle movements. Also during static stretching (SS) afferent activity is enhanced, but its effect on corticospinal excitability received limited attention and has only been investigated as a single average value spread over the entire stretching period. Using transcranial magnetic stimulation (TMS) the present study was conducted to explore the time course of corticospinal excitability during 30 seconds SS.

Motor evoked potentials (MEPs) after TMS were recorded from soleus (SOL) and tibialis anterior (TA) muscles in 14 participants during: a passive dynamic ankle dorsiflexion (DF), at six different time points during maximal individual SS $(3,6,9,18,21$ and 25 seconds into stretching), during a passive dynamic ankle plantar flexion (PF) and following SS.

During passive DF, MEPs amplitude was greater than baseline in both TA and SOL $(p=.001$ and $p=.005$ respectively). During SS, MEPs amplitude was greater than baseline in TA $(p=.006)$, but not in SOL. No differences between the investigated time points were found and no trend was detected throughout the stretching time. No effect in either muscle was observed during passive PF and after SS.

These results could suggest that an increased activity of secondary afferents from SOL muscle spindles exert a corticomotor facilitation on TA. The muscle-unspecific response observed during passive DF could instead be attributed to an increased activation within the sensorimotor cortical areas as a result of the awareness of the foot passive displacements.

\section{Introduction}

During the last few years, an increasing number of investigations tested the effects of stretching on spinal and corticospinal excitability, to explore whether the commonly observed stretching-induced decrease in physical performance can be attributed to neuromuscular factors ${ }^{1-5}$. Although some controversy seems to be resolved (for review ${ }^{6-8}$ ) and new light has been shed on alternative potential mechanisms ${ }^{9}$, a full picture is still lacking several key components, one of these being the limited information on the time course and instant values of the observed responses. Indeed, examining the neuromuscular effects of stretching by looking at an average value representative of a during- or an afterstretching phase, restricts the possibility to deeply understand the ongoing neurophysiological mechanisms ${ }^{10}$. This limitation is caused by two methodological requirements related to the assessment of spinal and corticospinal excitability, namely: having to collect and average several stimulations, and having to allow several seconds between one stimulation and the next ${ }^{11}$. As a result, the majority of the studies describe phenomena that do not represent instant points, but average values spread over a time window of several seconds. 
Acknowledging the limited ecological relevance of this issue when referring to sport and rehabilitation practices, from a strictly neurophysiological point of view, the possibility to detect a trend, or a specific time course of a response, may contribute to comprehend its origin and its implications. Moreover, it has to be considered that for measuring a parameter at the static end position of a stretch, and after it, a limb needs to be displaced to reach this position and then return from the stretching to a neutral position. Such lengthening and shortening displacements could influence the measurements collected during the stretch (following lengthening) and after the stretch (following shortening) ${ }^{12-17}$. One way to detect whether this has occurred, is by repeating the stretching protocol several times and thus also repeat several times the stimulation at specific time points into and after the stretch. This allows to distinguish between a potential long-lasting effect of the lengthening and shortening displacement, as well as to monitor the time course of the response throughout the stretching period. Such methodology, however, is not commonly adopted in studies about stretching. Only recently we implemented this approach to study in detail the time course of spinal excitability during and after stretching ${ }^{18,19}$ and the time course of corticospinal excitability following stretching ${ }^{19}$. However, to our knowledge, the behaviour of corticospinal excitability throughout the stretching time has never been investigated. On the contrary, using transcranial magnetic stimulation (TMS), corticospinal excitability has been studied during lengthening and shortening movements for over thirty years. Results for the arm and forearm muscles are generally consistent and demonstrate, with few exceptions ${ }^{20}$, a depression of corticospinal excitability during lengthening and an increase during both shortening passive movements ${ }^{13,16,17}$ and active contractions $12,17,21$. In the lower limb musculature, the results are more susceptible to the muscle tested, the contraction type and intensity, and the age of the participants ${ }^{22-26}$, but in general all these studies seem to indirectly confirm that variations in peripheral afferent input could affect pathway excitability 27,28 .

During static stretching (SS), the average TMS-induced motor evoked potentials (MEPs) amplitude has been reported to be reduced compared to baseline when the ankle joint was kept at $20^{\circ}$ dorsiflexion (DF), but not when it was kept at $10^{\circ} \mathrm{DF}^{29}$, which would suggest that: 1 ) the lengthening displacement, with related increased la afferent activity, does not have an effect lasting enough to influence spinal excitability during the following static stretching, and 2) that possibly also muscle spindle secondary afferents influence corticospinal excitability. However, it is still unknown whether the inhibition observed by Guissard and colleagues at $20^{\circ} \mathrm{SS}^{29}$ remained constant throughout the stretching time or showed a tendency to recover, as for example observed for spinal excitability ${ }^{18}$. Moreover, it is unknown whether the inhibition persists during the passive shortening phase back to the tested ankle neutral position and whether the passive plantarflexion (PF) contributes to the recovery or even promotes the facilitation detected within the first 2 seconds immediately after stretching ${ }^{19}$.

The current study was conducted to investigate the time course of corticospinal excitability during plantar flexors stretching. The passive lengthening and shortening phases were also monitored to obtain a clearer and more comprehensive picture. 


\section{Methods}

\section{Participants}

Fourteen recreational active individuals: 5 males (age $23.6 \pm 1.5$ years, body mass $75.2 \pm 7.3 \mathrm{~kg}$, stature $180 \pm 7.9 \mathrm{~cm}$ ) and 9 females (age $24.3 \pm 2.0$ years, body mass $58.0 \pm 3.0 \mathrm{~kg}$, stature $166 \pm 4.8 \mathrm{~cm}$ ) were recruited for this study. Volunteers abstain from any physical activity within $24 \mathrm{~h}$ before the testing. The study was approved by the Ethics Committee at the University of Graz (GZ. 39/77/63 ex 2013/14), all experiments were performed in accordance with relevant guidelines and regulations and written informed consent was obtained from all volunteers before the onset of the experimental procedures.

\section{Study design}

Participants attended the laboratory on two separate occasions: the first, lasting about 90 minutes, for familiarising with testing procedures and equipment and the second for the actual testing session. The testing session lasted about three hours.

The experiment consisted in the measurement of MEPs after TMS during: a passive dynamic ankle DF, at different time points during SS, during a passive dynamic ankle PF and following stretching.

Ten TMS stimulations were performed at each of the following time points into stretching: 3, 6, 9, 18, 21 and 25 seconds in random order. Moreover 16 MEPs were elicited during dynamic dorsiflexion and 14 during dynamic plantarflexion. Further stimulations were performed between 10 and 20 seconds at the end of the stretching procedure.

\section{Experimental procedures}

The experimental procedures have already described in our previous works ${ }^{18,19}$. Subjects were sitting on an isokinetic dynamometer (CON-TREX MJ, CMV AG, Duebendorf, Switzerland) with the standard setup for ankle joint rotation individually adjusted. Participants had their right knee fully extended and the foot resting on the dynamometer footplate, the ankle joint aligned with the dynamometer rotation shaft and the ankle angle set at $100^{\circ}\left(10^{\circ}\right.$ plantar flexion deviating from a neutral position at $\left.90^{\circ}\right)$. Volunteers sat with the trunk at $110^{\circ}$ and the head supported by a cushion (dentafix ${ }^{\circledR}$, pro medico HandelsGmbH, Graz, Austria) that once positioned could be deflated allowing the formation of a stable form molded on the volunteers' head and neck shapes. By using a remote control, the volunteers were instructed to adjust the dorsiflexion isokinetic rotation operated by the dynamometer around the foot plate until the point of perceived maximal DF. Participants were asked to keep their knee extended and to relax during the procedures.

Once the maximal individual dorsiflexion was defined, participants were prepared for surface electromyographic recording (EMG) from tibialis anterior muscle (TA) of both right and left leg as well as from the right soleus muscles (SOL). The volunteers then returned to sit on the dynamometer chair in the position described above and were instructed to relax meanwhile coil position and stimulation intensity 
for TMS was determined. Subsequently a trigger-driven sequence of stimulations started and continued for 150 seconds until 30 MEPs were collected for baseline reference values.

In order to perform all measurements at $100^{\circ}$ during dynamic $\mathrm{PF}$, following baseline recordings, the foot was passively rotated to a starting position of $110^{\circ}$ plantar flexion. In this way the stimulations during dorsiflexion movements $\left(20^{\circ} / \mathrm{s}\right)$ could be delivered when the ankle reached $100^{\circ} \mathrm{PF}$ (Figure 1 ).

The sequence of stimulations during the intervention is represented in Figure 1. A first stimulation was delivered during dynamic DF, then, once individual maximal dorsiflexion was reached, the position was kept for $30 \mathrm{~s}$ and during this period a second stimulation was delivered at one of the investigated time points $(3,6,9,18,21$ and $25 \mathrm{~s}$ into stretching). After this first $30 \mathrm{~s}$ SS bout, the foot was passively rotated $\left(20^{\circ} / \mathrm{s}\right)$ back to $110^{\circ} \mathrm{PF}$ and a third stimulation was delivered during the PF movement as the ankle was at $100^{\circ} \mathrm{PF}$. This procedure was repeated without pauses a second time with only one more stimulation delivered at maximal dorsiflexion and, after the second $30 \mathrm{~s}$ stretch, further stimulations were performed with the ankle joint back at $100^{\circ}$.

To collect a sufficient number of stimulations at each given time point, this $2 \times 30$ s stretching block was repeated 30 times; 50 second rest with the volunteer sitting were allowed between every block and two minutes rest with the volunteer standing up every 12 stretching applications.

\section{Surface electromyography}

Surface EMG was collected from the SOL and TA of the right leg. Additionally, the EMG signal was recorded from the tibialis anterior of the left leg to ensure the correct directional positioning of the coil. Electrodes (Blue Sensor N, Ambu A/S, Ballerup, Denmark) were placed in monopolar configuration for recording from the SOL muscle (as suggested by Hadoush et al. (2009)); the electrodes for TA were placed in bipolar configuration with an interelectrode distance of $20 \mathrm{~mm}$. One ground electrode per leg was placed over the tibial bone medial surface.

\section{Transcranial Magnetic Stimulation}

All stimulations at baseline, during dynamic DF and PF and after stretching were performed with the ankle joint at $100^{\circ} \mathrm{PF}$, stimulations during stretching were performed at individual maximal DF position (Figure 1).

Motor evoked potentials in response to single pulse TMS were induced by a Magstim 200, (Magstim Company Ltd., UK) using a double cone coil $(110 \mathrm{~mm}$ coil diameter). The coil was placed over the M1 of the leg area, 1-2 cm posterior from the vertex and slightly shifted to the left side in order to obtain the largest response from the contralateral right SOL. Resting motor threshold was determined as the minimum stimulator intensity able to evoke MEPs of at least $50 \mu \mathrm{V}$ amplitude in more than five out of ten consecutive trials ${ }^{31}$. To ensure a constant coil positioning throughout the experiments, subjects were wearing an electroencephalography cap on which the optimal coil position was marked with a soft pen. 
MEPs were elicited at 5-s intervals with stimulation intensity equal to $120 \%$ of the resting motor threshold.

\section{Data analysis}

Electromyography, foot displacement and trigger signals were synchronized (Dewetron 7.0 recording system), digitized with a sampling frequency of $10 \mathrm{KHz}$, stored on a PC and analysed using custom algorithms developed in Matlab (R2014b).

Baseline MEPs with peak to peak amplitude exceeding by \pm 2 standard deviations the average baseline value were discarded ${ }^{32}$. Similarly, consistency was checked within the 60 MEPs (10 stimulations, 6 time points) collected during stretching and the MEPs collected during DF and PF (16 and 14 MEPs respectively). Because voluntary activation increases MEP amplitude ${ }^{33}$, additional discards were applied in all those cases where MEPs were elicited in a pre-activation state of the target muscle (defined as EMG activity larger than $50 \mu \mathrm{V}$ in the 50 ms preceding the TMS pulse) ${ }^{34}$. All the remaining waves were retained and when a minimum number of 5 MEPs per data set was still available, the average value was used for statistical analysis.

\section{Statistical analysis}

Measurements were checked for normal distribution by Shapiro-Wilk test.

Friedman test followed by Wilcoxon tests with Bonferroni Holms adjustment was used to compare values at baseline, during dynamic DF, during SS (all values merged), during dynamic PF and post stretching.

The time course of MEPs amplitude throughout the stretching was tested by a Friedman test to compare values at different time points into stretching and further assessed with Pearson's correlation for linear regression analysis.

All statistical analysis was completed using PASW Statistic 18.0.0.

\section{Results}

Transcranial magnetic stimulation was well tolerated by all participants, stimulus intensity at resting motor threshold was $45 \pm 6 \%$ of the maximum stimulator output and no side-effects were reported. One subject was excluded for methodological problems. During SS, five volunteers showed pre-activation of the SOL muscle before several MEPs, consequently not a sufficient number of MEPs could be retained for subsequent analysis that was therefore performed on the remaining 8 participants. For TA no participants were excluded.

During static stretching the amplitude of the evoked MEPs was greater than at baseline for both SOL $\left(\chi^{2}{ }_{3}\right.$ $=16.393, p=.012)$ and TA $\left(\chi^{2}{ }_{3}=19.036, p=.004\right)$ at every tested time point for the TA, but only at 9,18 and 25 seconds into stretching for the SOL (at 3 and 6 seconds $p=.069$, at 21 seconds $p=.086$ ). No 
differences were observed between different data points into stretching (SOL: $\chi^{2}{ }_{3}=11.071, p=.05$ (no pair differences after Bonferroni Holms correction) and TA: $\chi^{2}{ }_{3}=7.714, p=.17$ ) and also no correlations between time into stretching and MEPs amplitude were detected for both SOL and TA $(p=.54$ and $p=.78$ respectively) (Figure 2). Consequently, all the MEPs elicited during stretching were averaged together.

Figure 3 shows MEPs data from a representative participant at baseline, during dynamic DF, during SS (random values over the stretching time), during dynamic PF and post stretching. As visible from the figure, for both SOL and TA, the amplitude of the MEPs evoked during the DF passive movement are clearly bigger compared to those recorded at the other stimulation points (Figure 3). Group average values (presented as percentage of variation from baseline values in Figure 4) reflect the data of the representative participant. There was a statistically significant difference between the amplitude of the MEPs evoked in the different conditions for both SOL $\left(\chi^{2}{ }_{3}=22.489, p=0.00016\right)$ and TA $\left(X^{2}{ }_{3}=30.533, p\right.$ $=.000004)$ with higher MEPs amplitude during dynamic DF compared to baseline for both muscles ( $Z=$ $-2.830, p=.005$ and $Z=-3.180, p=.001$, for SOL and TA respectively). Additional pair comparisons remained statistically significant after Bonferroni Holms correction for TA (DS vs post: $p=.001$, DF vs PF: $p=.002$ and SS vs baseline: $p=.006)$, but not for SOL.

\section{Discussion}

The aim of the present study was to examine the time course of corticospinal excitability during SS of the plantar flexors as well as to monitor potential variation during dynamic DF and PF. For both SOL and TA, the amplitude of the MEPs evoked during DF was significantly higher compared to baseline. Throughout the entire $30 \mathrm{sec}$ SS the corticomotor excitability was significantly increased for TA and did not show a time-dependent trend.

\section{Response modulation during SS}

During the 30 seconds SS, MEPs were collected at six time points: $3,6,9,18,21$ and 25 seconds into stretching. Since we observed a clear facilitation during the DF movement required to bring the foot to maximal individual static DF position, we could have expected to capture a long-lasting effect within the first measurement at 3 seconds. Looking at Figure 2 this seems to be confirmed in most of the subjects, and from the group average it is immediately visible a pattern consisting in a sharp facilitation at 3 seconds into stretching, a reduction of this from 3 to 6 seconds, followed by a more or less linear increase. Both muscles showed the same behaviour, although no differences between time points into stretching were observed. Interestingly, such behaviour appears specular to the H-reflex inhibition we previously observed ${ }^{18}$. Figure 5 was constructed with the data of H-reflexes in a previous experiment ${ }^{18}$. The group average $96 \%$ increase in MEPs size at 3 seconds is matched by a $59 \%$ decrease in H-reflex, from 3 to 6 seconds MEPs size decreased whilst H-reflex increased.

This symmetry, that ends from 6 seconds on, seems again to support the hypothesis of a relationship between variation in peripheral afferent input and corticospinal excitability and specifically suggests that 
increased la afferents from SOL muscle spindles, are responsible for facilitation on corticospinal excitability of its antagonist TA. This crossed agonist/antagonist effect has already been shown by Bertolasi and colleagues ${ }^{35}$ who demonstrated an effect of forearm flexor muscle afferents on excitability of corticospinal projections to antagonist muscles. Although the authors reported an inhibitory action to the antagonist, it has to be considered the relevance of the adopted conditioning-test intervals ${ }^{27}$ : in Bertolasi et al. ${ }^{35}$ it was circa 20 ms whilst a minimum 500 ms elapsed from the beginning of the stretch to the TMS stimulation in our study. Moreover, the stimulus was not a short electrical input, but a prolonged passive displacement. Thus, we can hypothesise that afferent projections from one muscle have a crossed effect on the cortical excitability of its agonist, but whether this has an inhibitory or facilitatory effect is in relation to several factors including stimulus duration/modality, conditioning-test interval and muscles tested.

From 3 seconds into stretching, the la activity can be expected to be markedly reduced, whilst firing frequency of muscle spindle secondary afferents (group II) increases. Afferents from SOL secondary endings seem to affect TA corticospinal excitability in a similar, although weaker, way (Figure 4B). Increased activity from secondary muscle spindle afferents can depress Renshaw cells recurrent inhibition, as demonstrated both on decerebrated cats ${ }^{36}$ and through computer modelling ${ }^{37}$, resulting therefore in the observed facilitation.

\section{Response modulation during dynamic DF}

The observed corticomotor facilitation during DF was registered for both the elongated (SOL) and the shortened (TA) muscles. While the increased corticomotor excitability of TA during DF is in line with studies showing increased MEP during passive muscle shortening $12,13,16,17,21,25$, the simultaneously recorded facilitation in the elongated muscle SOL is not supported by previous findings, which commonly report reduced MEP amplitude during lengthening of the wrist and elbow flexors and extensors $12,13,16,17,21,38$. However, differently to the upper limb, responses in the lower limb are sometimes contradictory being susceptible to more variables ${ }^{22-26}$. For example Škarabot and colleagues reported a facilitation in the shortened TA in young ${ }^{25}$ but not in old participants ${ }^{26}$ and no effect on the lengthened SOL ${ }^{25,26}$, whilst Hultborn and colleagues ${ }^{20}$ did not report any facilitation in neither TA nor SOL, and in the present study we witnessed a facilitation in both TA and SOL.

On the origin of the facilitation we observed, potential sites of modulation of the MEP in response to TMS include intrinsic cortical inputs to the pyramidal tract neurons in M1, the activity of the a-motoneurons and interneuron network at spinal level and the afferent signals arising from activated sensory receptors. Considering that TMS activates predominantly monosynaptic pathways to the a-motoneurons and these connections are not exposed to presynaptic inhibition ${ }^{13}$, it seems that the observed excitability alterations during DF can be attributed either to cortical inputs to the corticospinal pathways or to postsynaptic inputs directly modulating the a-motoneurons. However, a recent study showed that during passive ankle movements, the excitability at the lumbar spinal segmental level was not modulated in 
neither TA nor SOL ${ }^{25}$, suggesting supraspinal rather than postsynaptic contributions to the observed MEP facilitation.

Supraspinal structures can indeed be affected by proprioceptive afferents. During passive lengthening movements, la fibres from muscle spindles respond to changes in muscle length by increasing their firing rate ${ }^{39,40}$. The respective inflows projecting primarily to the area $3 a$ in the primary somatosensory cortex (S1) ${ }^{41}$, can activate indirectly the cortical inputs to the pyramidal tract neurons in M1. Such co-activation within the S1 area and the motor areas (M1 and SMA) in response to passive proprioceptive stimulation (passive fingers' flexion and extension) was demonstrated in a fMRI study ${ }^{42}$. It is known that the finely scaled topographic maps of S1 and M1 enable both areas to have highly specialized responses to changes in the periphery ${ }^{43,44}$. As shown in a sensorimotor slice ${ }^{45}$, the anatomical and functional sensorimotor connections are reciprocal and their localization in cortical layers $\mathrm{V}$ and $\mathrm{VI}$ allow descending outputs also to the spinal cord ${ }^{46}$. In our study we can expect that the increase in la afferents activity from SOL during DF reached the somatosensory area (3a), which transmits these inputs further to the M1 output neurons, increasing the excitability of the descending corticomotor pathway.

Such increased excitability originating from the cortex with related facilitation on the descending drive, could be a compensation mechanism for the spinal inhibition (typically observed as decrease in H-reflex during lengthening movements for review see ${ }^{10}$ ), as shown in a study with active muscle lengthening ${ }^{21}$. However, if an increased la activity provoked an increase in corticospinal excitability or reduced cerebellocortical inhibition on projections on the homonymous muscle, we should have observed not only greater MEPs in the SOL during DF, but also in the TA during PF movement. Alternatively, in case of projections on the antagonist muscles, we should have observed greater MEPs in the TA during DF together with greater MEPs in the SOL during PF movement, but neither of these two scenarios was the case (Figure 4).

A possible explanation of this not muscle-specific response could be attributed to the awareness of the subjects of the foot passive displacements, that, for methodological requirements, was repeated 60 times. Indeed, in healthy individuals, passive movements are shown to activate not only the primary somatosensory cortex but also the primary motor cortex, supplementary motor area, and posterior parietal cortex as well as the secondary somatosensory cortex (S2) (for review see ${ }^{47}$ ). Moreover, passive movements can selectively increase the cortical excitability dependent on the duration and velocity of movements, the presence of rest, but also on whether attention was directed to the movement. Attention to the stimulated side during an intervention (peripheral afferent stimulation, passive movements) decreases the activity of the inhibitory cortical circuits, and thus increases corticospinal excitability. In our study the subjects were instructed to avoid observing the stretching extremity. Nevertheless, the effect of attention to the movement throughout the experimental procedure cannot be completely excluded. In addition, passive movements repeated with the same amplitude and velocity for a certain time might be able to induce attempted movement, thereby activating neurons in $\mathrm{M} 1{ }^{48}$ and resulting in increased MEPs during DF for both TA and SOL muscles. 
Despite the precise mechanisms responsible for the increased excitability cannot be clarified with this study, the findings may have clinical potential in neurorehabilitation, where passive movements are commonly applied in physiotherapy. For example in patients with paresis of the lower extremity passive dorsi- and plantar flexions can be repeatedly performed using robotic devices. It is expected that the augmented proprioceptive input facilitating motor cortical excitability, may promote motor activation and thus can improve motor recovery.

\section{Limitations}

In the present study M-max were only recorded from the SOL. Therefore MEP/M-max normalisation would be only possible for this muscle. However, M-max is known not to be affected during and after stretching, therefore we could normalise our data by expressing them as a percentage of variation from baseline values.

Since the stretching protocol and respectively the stimulations at specific time points into and after the stretch had to be repeated several times, the TMS hot spot was located for stimulating the SOL, and we did not perform separate stimulations targeting specifically the TA. Nevertheless, we could observe differential MEP changes also in TA.

Finally, the inevitable methodological requirement of repeating the stretching procedure several times, could have influeced muscle stiffness with related consequences for muscle afferent feedback and proprioception.

In conclusion, it was shown that passive dynamic dorsiflexion of the plantar flexors and their static stretching facilitated the MEP after TMS recorded from both, the stretched and the shortened muscles.

This faciliation did not show a time-dependent trend or a tendency to recover during the stretching period.

\section{Declarations}

The authors declare that they have no conflict of interest.

Data will be made available on request

Funding: Austrian Science Fund (FWF), Grant/Award Number: 'P 27665'.

\section{References}

1. Pulverenti, T. S., Trajano, G. S., Kirk, B. J. \& Blazevich, A. J. The loss of muscle force production after muscle stretching is not accompanied by altered corticospinal excitability. Eur. J. Appl. Physiol. 119, 2287-2299 (2019). 
2. Pulverenti, T. S., Trajano, G. S., Walsh, A., Kirk, B. J. \& Blazevich, A. J. Lack of cortical or la-afferent spinal pathway involvement in muscle force loss after passive static stretching. J. Neurophysiol. 123, 1896-1906 (2020).

3. Pulverenti, T. S., Trajano, G. S., Kirk, B. J., Bochkezanian, V. \& Blazevich, A. J. Plantar flexor muscle stretching depresses the soleus late response but not tendon tap reflexes. Eur. J. Neurosci. 53, 31853198 (2021).

4. Cè, E. et al. Neuromuscular versus Mechanical Stretch-induced Changes in Contra-versus Ipsilateral Muscle. Med. Sci. Sports Exerc. (2020).

5. Coratella, G. et al. Neuromuscular Correlates of the Contralateral Stretch-induced Strength Loss. Med. Sci. Sports Exerc. (2021).

6. Chaabene, H., Behm, D. G., Negra, Y. \& Granacher, U. Acute effects of static stretching on muscle strength and power: An attempt to clarify previous caveats. Front. Physiol. 10, 1468 (2019).

7. Behm, D. G., Kay, A. D., Trajano, G. S. \& Blazevich, A. J. Mechanisms underlying performance impairments following prolonged static stretching without a comprehensive warm-up. Eur. J. Appl. Physiol. 1-28 (2020).

8. Trajano, G. S. \& Blazevich, A. J. Static Stretching Reduces Motoneuron Excitability: The Potential Role of Neuromodulation. Exerc. Sport Sci. Rev. 49, 126-132 (2021).

9. Trajano, G. S., Taylor, J. L., Orssatto, L. B., McNulty, C. R. \& Blazevich, A. J. Passive muscle stretching reduces estimates of persistent inward current strength in soleus motor units. J. Exp. Biol. 223, jeb229922 (2020).

10. Budini, F. \& Tilp, M. Changes in H-reflex amplitude to muscle stretch and lengthening in humans. Rev. Neurosci. 27, 511-522 (2016).

11. Knikou, M. The H-reflex as a probe: pathways and pitfalls. J. Neurosci. Methods 171, 1-12 (2008).

12. Abbruzzese, G., Morena, M., Spadavecchia, L. \& Schieppati, M. Response of arm flexor muscles to magnetic and electrical brain stimulation during shortening and lengthening tasks in man. J. Physiol. 481, 499-507 (1994).

13. Lewis, G. N., Byblow, W. D. \& Carson, R. G. Phasic modulation of corticomotor excitability during passive movement of the upper limb: effects of movement frequency and muscle specificity. Brain Res. 900, 282-294 (2001).

14. Sekiguchi, H., Kimura, T., Yamanaka, K. \& Nakazawa, K. Lower excitability of the corticospinal tract to transcranial magnetic stimulation during lengthening contractions in human elbow flexors. Neurosci. Lett. 312, 83-86 (2001).

15. Sekiguchi, H., Nakazawa, K. \& Suzuki, S. Differences in recruitment properties of the corticospinal pathway between lengthening and shortening contractions in human soleus muscle. Brain Res. 977, 169-179 (2003).

16. Coxon, J. P., Stinear, J. W. \& Byblow, W. D. Amplitude of muscle stretch modulates corticomotor gain during passive movement. Brain Res. 1031, 109-117 (2005). 
17. Chye, L., Nosaka, K., Murray, L., Edwards, D. \& Thickbroom, G. Corticomotor excitability of wrist flexor and extensor muscles during active and passive movement. Hum. Mov. Sci. 29, 494-501 (2010).

18. Budini, F., Christova, M., Gallasch, E., Rafolt, D. \& Tilp, M. Soleus H-Reflex Inhibition Decreases During 30 s Static Stretching of Plantar Flexors, Showing Two Recovery Steps. Front. Physiol. 9, (2018).

19. Budini, F. et al. Transient increase in cortical excitability following static stretching of plantar flexor muscles. Front. Physiol. 9, 530 (2018).

20. Hultborn, H. et al. On the mechanism of the post-activation depression of the H-reflex in human subjects. Exp. Brain Res. 108, 450-462 (1996).

21. Gruber, M., Linnamo, V., Strojnik, V., Rantalainen, T. \& Avela, J. Excitability at the motoneuron pool and motor cortex is specifically modulated in lengthening compared to isometric contractions. $J$. Neurophysiol. 101, 2030-2040 (2009).

22. Doguet, V. et al. Muscle length effect on corticospinal excitability during maximal concentric, isometric and eccentric contractions of the knee extensors. Exp. Physiol. 102, 1513-1523 (2017).

23. Duclay, J., Pasquet, B., Martin, A. \& Duchateau, J. Specific modulation of corticospinal and spinal excitabilities during maximal voluntary isometric, shortening and lengthening contractions in synergist muscles. J. Physiol. 589, 2901-2916 (2011).

24. Duclay, J., Pasquet, B., Martin, A. \& Duchateau, J. Specific modulation of spinal and cortical excitabilities during lengthening and shortening submaximal and maximal contractions in plantar flexor muscles. J. Appl. Physiol. 117, 1440-1450 (2014).

25. Škarabot, J. et al. Corticospinal excitability of tibialis anterior and soleus differs during passive ankle movement. Exp. Brain Res. 237, 2239-2254 (2019).

26. Škarabot, J., Ansdell, P., Howatson, G., Goodall, S. \& Durbaba, R. Corticospinal responses during passive shortening and lengthening of tibialis anterior and soleus in older compared to younger adults. Exp. Physiol. 105, 419-426 (2020).

27. Chen, R., Corwell, B. \& Hallett, M. Modulation of motor cortex excitability by median nerve and digit stimulation. Exp. Brain Res. 129, 77-86 (1999).

28. Trompetto, C., Buccolieri, A. \& Abbruzzese, G. Intracortical inhibitory circuits and sensory input: a study with transcranial magnetic stimulation in humans. Neurosci. Lett. 297, 17-20 (2001).

29. Guissard, N., Duchateau, J. \& Hainaut, K. Mechanisms of decreased motoneurone excitation during passive muscle stretching. Exp. Brain Res. 137, 163-169 (2001).

30. Hadoush, H. et al. Monopolar surface electromyography: a better tool to assess motoneuron excitability upon passive muscle stretching. J. Physiol. Sci. 59, 243-247 (2009).

31. Rossini, P. M. et al. Non-invasive electrical and magnetic stimulation of the brain, spinal cord and roots: basic principles and procedures for routine clinical application. Report of an IFCN committee. Electroencephalogr. Clin. Neurophysiol. 91, 79-92 (1994).

32. Nogueira-Campos, A. A. et al. Corticospinal excitability preceding the grasping of emotion-laden stimuli. PloS One 9, e94824 (2014). 
33. Lazzaro, V. D. et al. Effects of voluntary contraction on descending volleys evoked by transcranial stimulation in conscious humans. J. Physiol. 508, 625-633 (1998).

34. Ter Braack, E. M., de Goede, A. A. \& van Putten, M. J. Resting motor threshold, MEP and TEP variability during daytime. Brain Topogr. 32, 17-27 (2019).

35. Bertolasi, L., Priori, A., Tinazzi, M., Bertasi, V. \& Rothwell, J. C. Inhibitory action of forearm flexor muscle afferents on corticospinal outputs to antagonist muscles in humans. J. Physiol. 511, 947956 (1998).

36. Fromm, C., Haase, J. \& Wolf, E. Depression of the recurrent inhibition of extensor motoneurons by the action of group II afferents. Brain Res. 120, 459-468 (1977).

37. Graham, B. P. \& Redman, S. J. Dynamic behaviour of a model of the muscle stretch reflex. Neural Netw. 6, 947-962 (1993).

38. Lewis, G. N. \& Byblow, W. D. Modulations in corticomotor excitability during passive upper-limb movement: is there a cortical influence? Brain Res. 943, 263-275 (2002).

39. Day, J., Bent, L. R., Birznieks, I., Macefield, V. G. \& Cresswell, A. G. Muscle spindles in human tibialis anterior encode muscle fascicle length changes. J. Neurophysiol. 117, 1489-1498 (2017).

40. Macefield, V. G. \& Knellwolf, T. P. Functional properties of human muscle spindles. J. Neurophysiol. 120, 452-467 (2018).

41. Yamada, H. et al. Representation of afferent signals from forearm muscle and cutaneous nerves in the primary somatosensory cortex of the macaque monkey. PLoS One 11, e0163948 (2016).

42. Nasrallah, F. A. et al. Functional connectivity of brain associated with passive range of motion exercise: Proprioceptive input promoting motor activation? Neurolmage 202, 116023 (2019).

43. Brooks, V., Rudomin, P. \& Slayman, C. Peripheral receptive fields of neurons in the cat's cerebral cortex. J. Neurophysiol. 24, 302-325 (1961).

44. Li, C.-X. \& Waters, R. S. Organization of the mouse motor cortex studied by retrograde tracing and intracortical microstimulation (ICMS) mapping. Can. J. Neurol. Sci. 18, 28-38 (1991).

45. Rocco, M. M. \& Brumberg, J. C. The sensorimotor slice. J. Neurosci. Methods 162, 139-147 (2007).

46. Hoffer, Z. S., Arantes, H. B., Roth, R. L. \& Alloway, K. D. Functional circuits mediating sensorimotor integration: quantitative comparisons of projections from rodent barrel cortex to primary motor cortex, neostriatum, superior colliculus, and the pons. J. Comp. Neurol. 488, 82-100 (2005).

47. Onishi, H. Cortical excitability following passive movement. Phys. Ther. Res. R0001 (2018).

48. Truccolo, W., Friehs, G. M., Donoghue, J. P. \& Hochberg, L. R. Primary motor cortex tuning to intended movement kinematics in humans with tetraplegia. J. Neurosci. 28, 1163-1178 (2008).

\section{Figures}




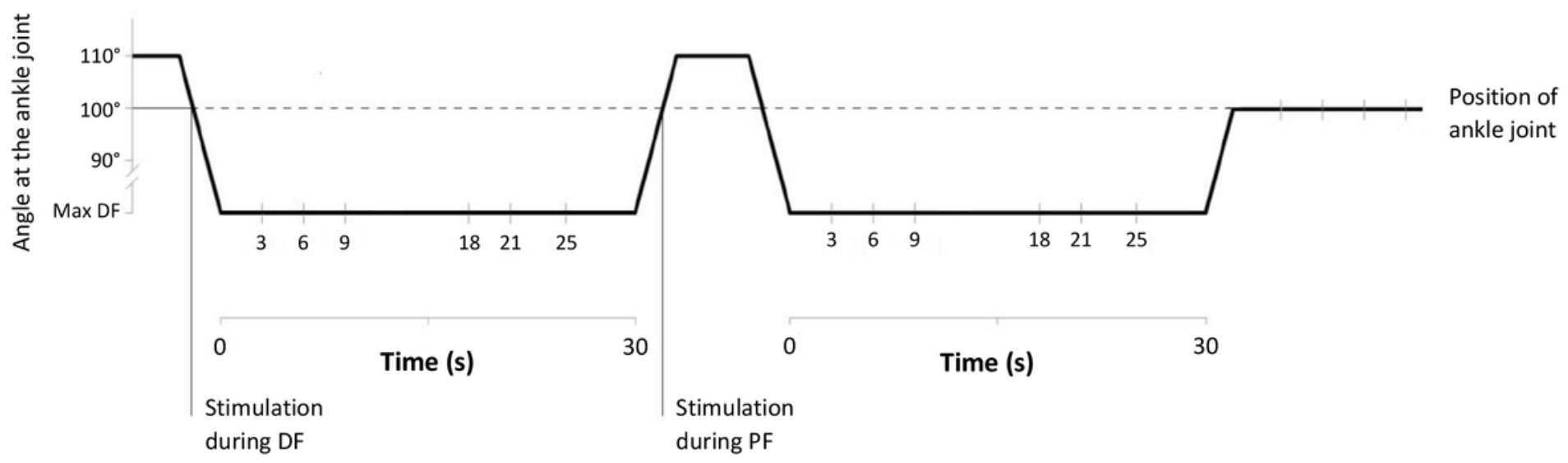

Figure 1

Stretching protocol and stimulation points 


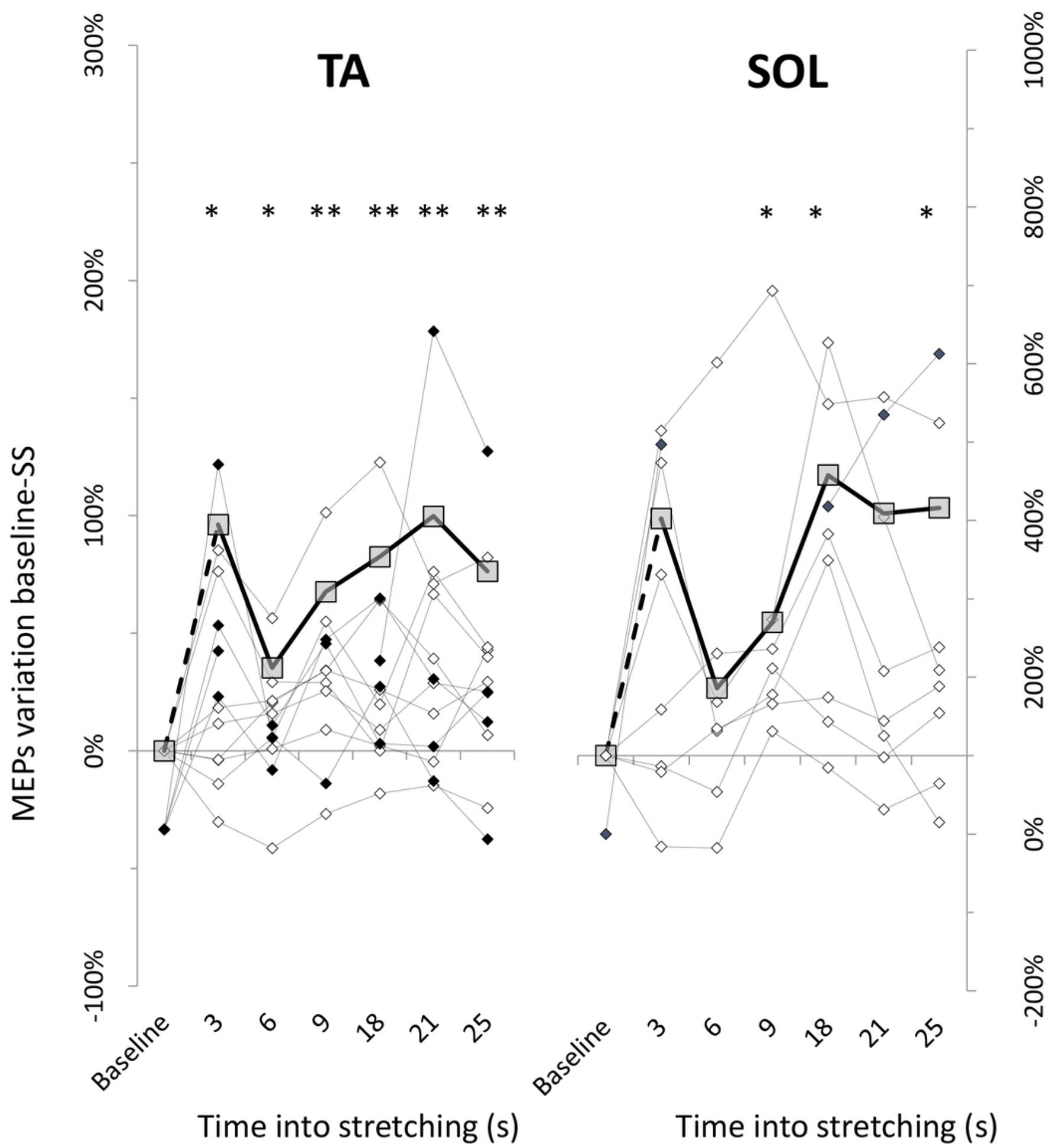

Figure 2

Time course of MEPs facilitation during stretching MEPs at each tested time point during stretching Group average (thick line with squares) and individual (thin dotted line with diamonds) are expressed as percentage of variation from baseline values. On the secondary axis are plotted individual subjects represented with filled diamonds 

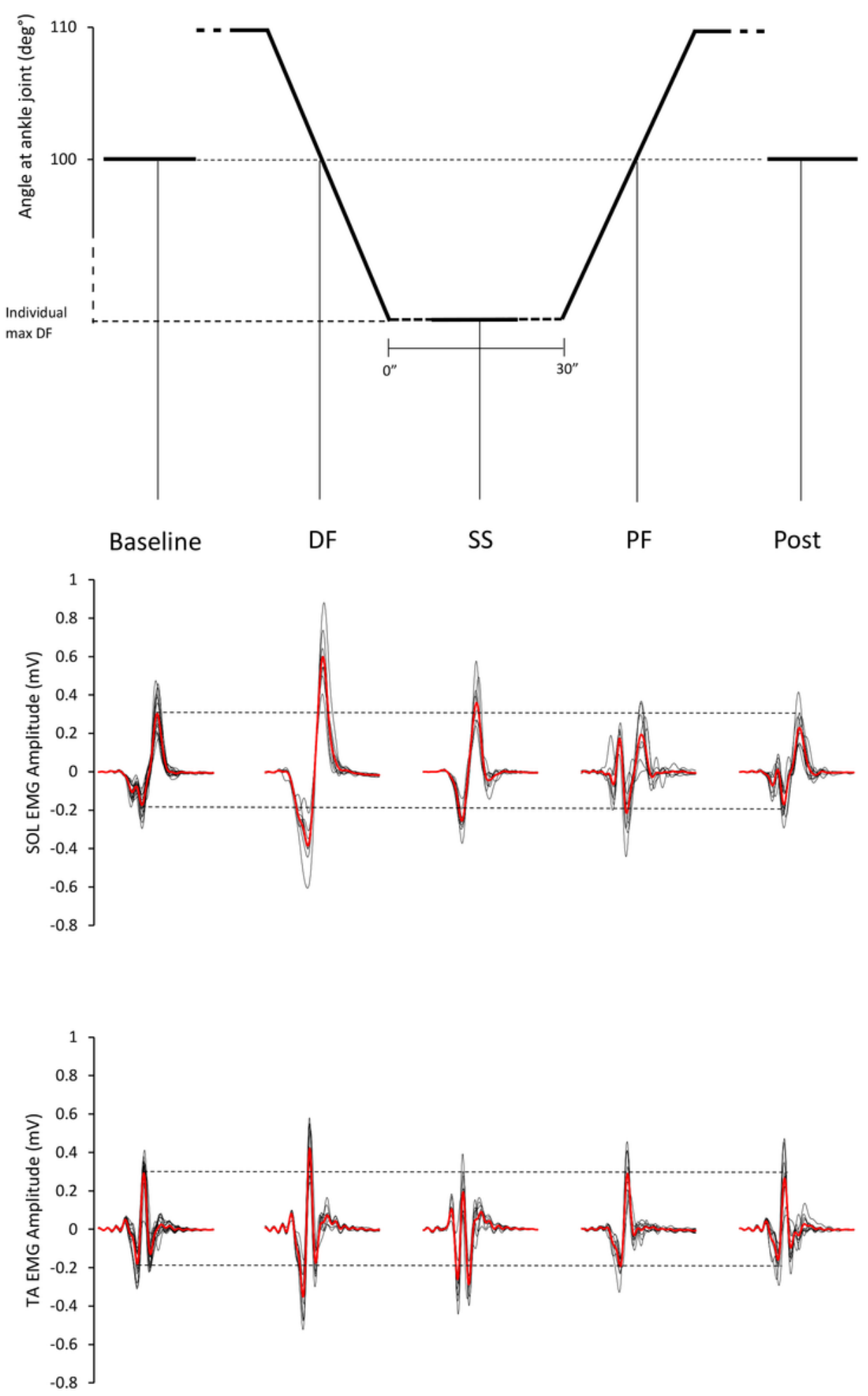

\section{Figure 3}

single subject example for MEPs On the upper part of the graph is outlined the angle at the ankle joint at which the stimulations were delivered. In the middle and lower part of the graph are presented the SOL and TA EMG tracks recorded at: baseline, during DF, during SS, during PF and after stretching. For baseline circa 15 waves are superimposed (black waves), for all the other stimulation points circa 10 waves are superimposed. The thicker red waves represent the average values and the dashed horizontal 
lines limit the positive and negative peak of the average baseline value. For graphical purposes raw data was down sampled and low passed filtered. Comparison to Baseline: * $p<.05 ; * \star p<.01$

A

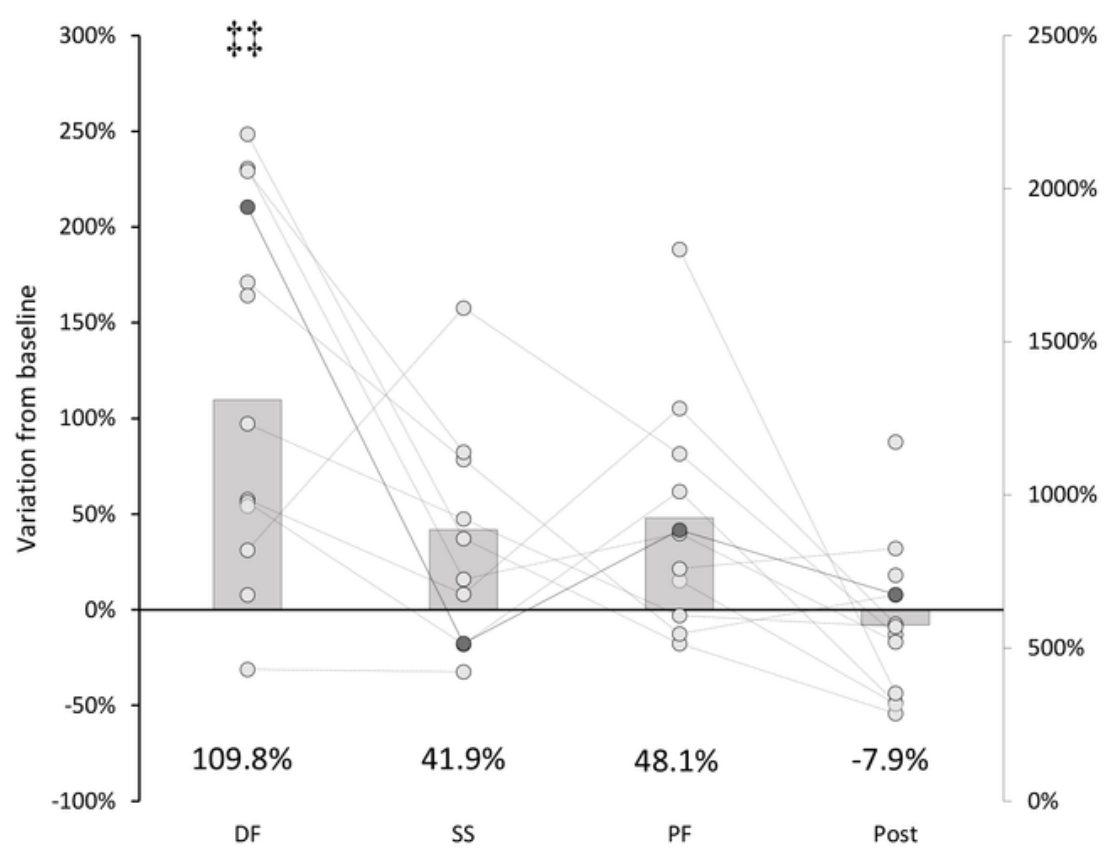

B

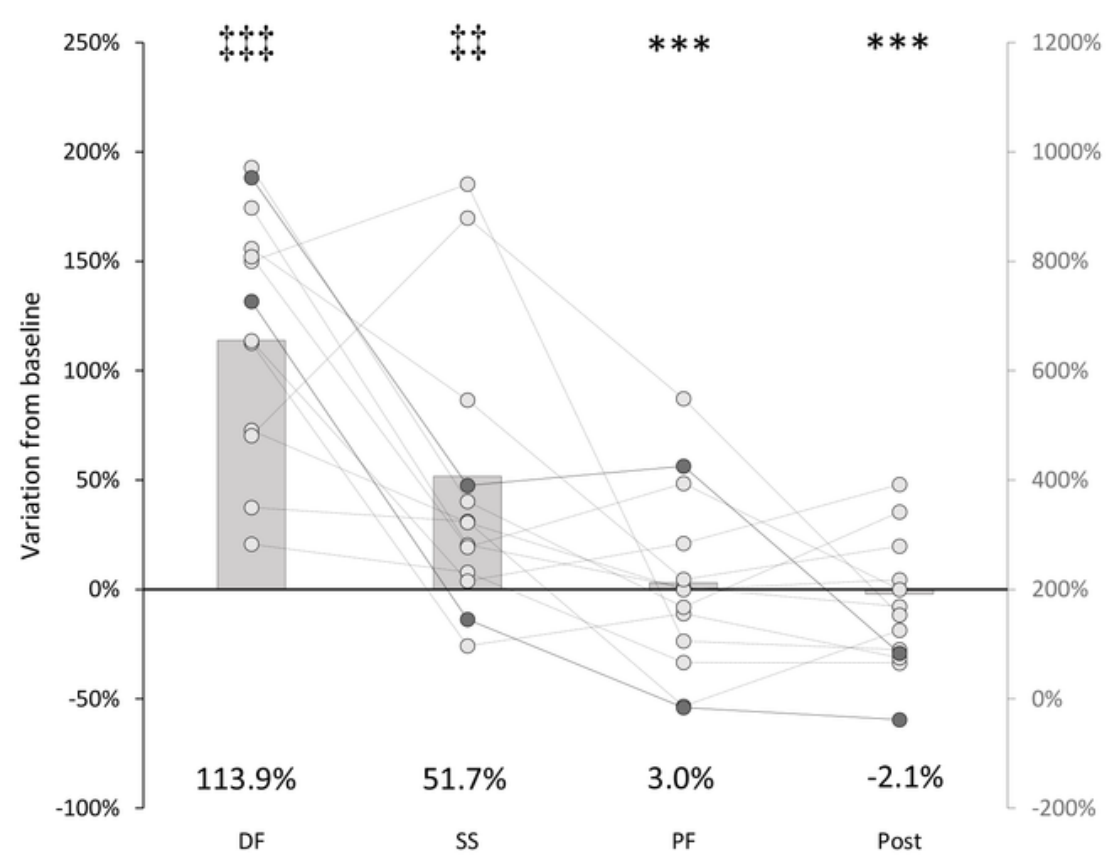

Figure 4

A: SOL; B: TA On the primary axis, bars: group average MEPs amplitude expressed as percentage of baseline values (with data labels); connected dots: invidividual values. On the secondary axis are plotted the individual subjects represented with thicker lines and filled circles (for graphical reasons, removed 
from the group average bars). Comparison to DF: $\star \star \star ~ p<.005 ;$ Comparison to baseline: 㧊 $p<.01$; 执 $p$ $<.001$

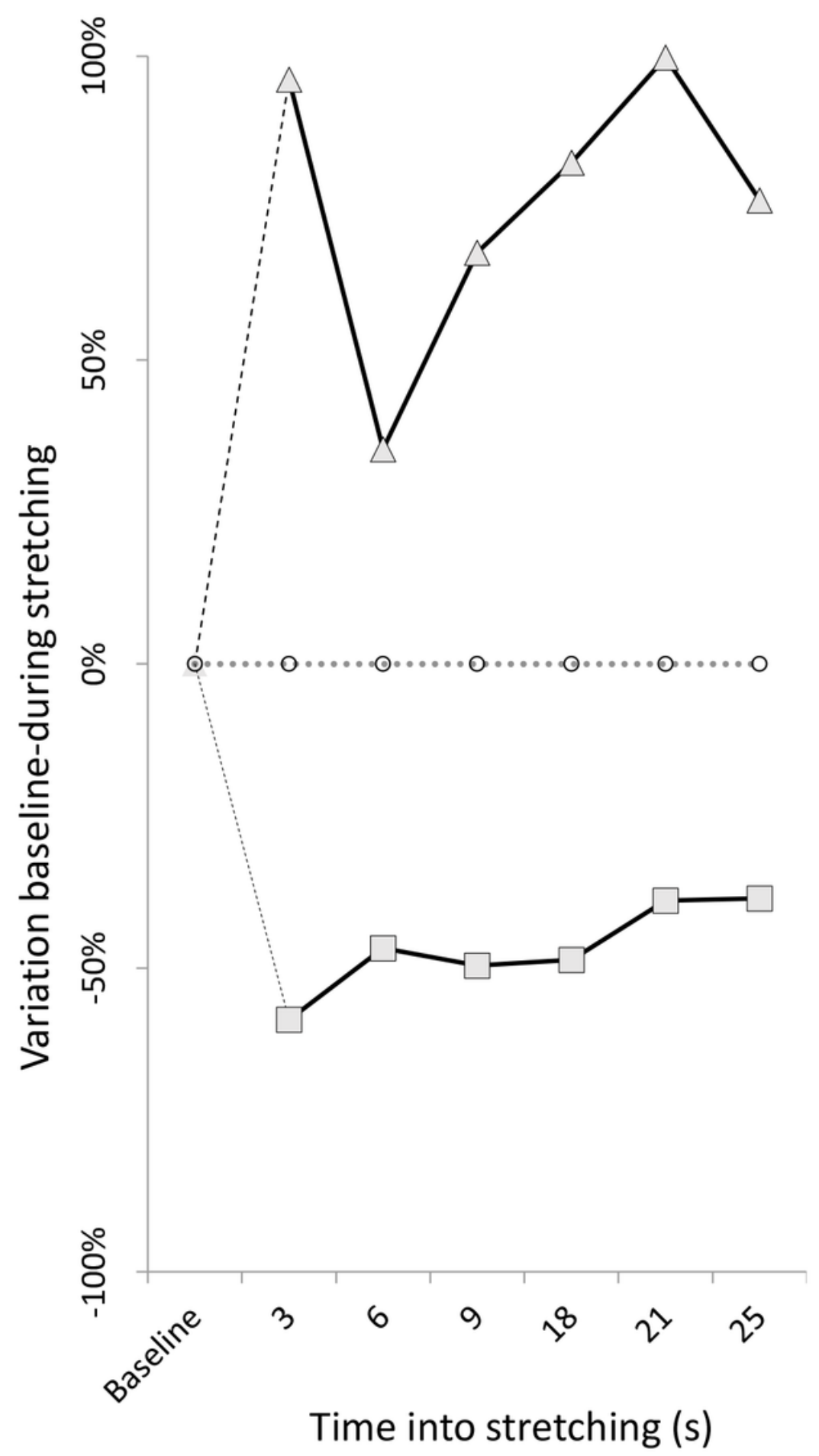

Figure 5

Time course of TA MEPs facilitation (upper line with triangles) and SOL H-reflex inhibition (lower line with squares) during stretching. SOL H-reflex data from 18. 